\title{
Acute Abdomen in Woman of Childbearing Age: Appendicitis or Pelvic Inflammatory Disease? A Systematic Review
}

\author{
Perrone Gennaro*1, Petracca Gabriele Luciano ${ }^{1}$, Prioriello Concetta ${ }^{2}$, Morini Andrea ${ }^{2}$, Bonati \\ Elena $^{2}$, Disisto Caterina ${ }^{1}$, Giuffrida Mario $^{2}$, Tarasconi Antonio ${ }^{1}$, Coccolini Federico ${ }^{3}$, Sartelli \\ Massimo $^{4}$, Ansaloni Luca ${ }^{5}$, Livrini Marco ${ }^{1}$ and Catena Fausto ${ }^{1}$ \\ ${ }^{1}$ Department of Emergency and Trauma Surgery, University Hospital of Parma, Italy
}

${ }^{2}$ Unit of General Surgery, Department of Medicine and Surgery, University Hospital of Parma, Italy

${ }^{3}$ General, Emergency and Trauma Surgery, Pisa University Hospital, Italy

${ }^{4}$ Department of General Surgery, Macerata Hospital, Italy

${ }^{5}$ Department of Surgery, University Hospital of San Matteo Pavia, Italy

*Corresponding author: Perrone Gennaro, Department of Emergency and Trauma Surgery, University Hospital of Parma, Italy

\begin{abstract}
ARTICLE INFO
Received: 幽 May 01, 2021

Published: 慧 May 10, 2021

Citation: Perrone Gennaro, Petracca Gabriele Luciano, Prioriello Concetta, Morini Andrea, Bonati Elena, et al. Acute Abdomen in Woman of Childbearing Age: Appendicitis or Pelvic Inflammatory Disease? A Systematic Review. Biomed J Sci \& Tech Res 35(4)-2021. BJSTR. MS.ID.005738.
\end{abstract}

Abbreviations: US: Ultrasound; CT: Computer Tomography; CRP: C-Reactive Protein; AIR: Appendicitis Inflammatory Response; AAS: Adult Appendicitis Score; PID: Pelvic Inflammatory Disease; PMN: Polymorphonuclear; NAATs: Nucleic Acid Amplification Tests

\section{ABSTRACT}

Acute abdomen in women of childbearing age is a clinical presentation that requires emergency surgical treatment in many cases and rapid diagnosis is oftentimes essential. Most are the causes of acute abdomen: gynecological (ectopic pregnancy, ovarian torsion, ovarian cysts, endometriosis, pelvic inflammatory disease) and non-gynecological (appendicitis, Crohn's disease, Meckel's diverticulitis, sigmoid diverticulitis, perforated peptic ulcer, viral gastroenteritis, hernias, urological disease). Appendicitis and pelvic inflammatory disease (PID) are two of the most common causes of abdominal pain in women of childbearing age. This article is a systematic review of literature of the differential diagnosis between appendicitis and PID. The diagnosis is clinical, laboratory and radiological. When the sign and symptoms and the biochemical markers are nonspecific abdominal ultrasound (US) is the first radiological test that is performed, but the computer tomography (CT) remains the best specific and sensible exam for the differential diagnosis. Nuclear Magnetic Resonance is performed when CT is contraindicated. Other test like transvaginal ultrasound is a relatively simple and inexpensive diagnostic test that could easily complement the classic abdominal US and be part of the routine diagnostic protocol of women with acute abdominal pain. Therefore, a careful management of the patient starting from the clinical examination and passing through the laboratory and radiological characteristics is necessary.

Keywords: Acute Abdomen; Right Acute Abdomen; Appendicitis; Pelvic Inflammatory Disease; Women of Childbearing Age

\section{Introduction}

Acute abdomen is a clinical presentation that requires emergency surgical treatment in many cases, rapid diagnosis is oftentimes essential. The diagnoses associated with an acute abdomen vary according to age and gender [1], and it is more difficult in young women, in which it is necessary to distinguish between gynecological and non-gynecological diseases (Table 1). Non-gynecological causes include appendicitis, which remains one of the main causes of acute abdomen in childbearing women. The diagnosis of acute appendicitis is essentially clinical. Appendicitis can initially present with periumbilical pain that migrates to the right 
lower quadrant, classically more intense over McBurney's point. The pain can be associated with fevers along with nausea, vomiting and anorexia, that classically occur after pain onset. Common laboratory abnormalities include leukocytosis (white blood cells WBS $>20.000$ cells $/ \mathrm{mm}^{3}$ ) and an elevated C-reactive protein (CRP) level. Although appendicitis is a highly prevalent disease, especially in Western countries, diagnosis remains a challenge for the surgeon and requires a combination of medical history, laboratory tests, clinical and diagnostic imaging (ultrasound, CT and MRI). Several clinical and laboratory-based scoring systems have been devised to assist diagnosis and the most widely used are the Alvarado score, the AIR (Appendicitis Inflammatory Response) and the AAS (Adult Appendicitis Score).

Table 1.

\begin{tabular}{|c|c|}
\hline \multicolumn{2}{|c|}{ Lower Quadrant Abdominal Pain in Young Women } \\
\hline Non-Gynaecological Disease & Gynaecological Disease \\
\hline $\begin{array}{l}\text { - Appendicitis } \\
\text { - Crohn's disease } \\
\text { - Meckel's diverticulitis } \\
\text { - Sigmoid diverticulitis } \\
\text { - Perforated peptic ulcer } \\
\text { - Viral gastroenteritis } \\
\text { - Mesenteric adenitis } \\
\text { - Ventral and inguinal hernias } \\
\text { - Urological diseases: } \\
\text { - Pyelonephritis } \\
\text { - Perinephric abscess } \\
\text { - Urolithiasis } \\
\text { - Urinary tract infections }\end{array}$ & $\begin{array}{l}\text { - Ectopic pregnancy } \\
\text { - Ruptured follicular or corpus luteum } \\
\text { cust } \\
\text { - Ovarian torsion } \\
\text { - PID } \\
\text { - Haemorrhage of an ovarion cyst } \\
\text { - Mittelschmera } \\
\text { - Endometriosis }\end{array}$ \\
\hline
\end{tabular}

The last WSES guidelines [2] on appendicitis recommend the use of AIR score and AAS score as clinical predictors of acute appendicitis (Table 2). In women of childbearing age, the diagnosis is further complicated because, in case of lower abdominal pain, appendicitis must be distinguished from a variety of gynecological conditions in order to avoid unnecessary intervention. A careful gynecological history should be taken in all women with acute abdomen, concentrating on menstrual cycle, vaginal discharge and possible pregnancy. For all female patients of childbearing age, pregnancy testing should always be part of the workup for any abdominal pain to rule out ectopic pregnancy. Other gynecologic causes include ruptured ovarian cysts, mittelschmerz (midcycle pain occurring with ovulation), endometriosis, ovarian torsion, ectopic pregnancy and pelvic inflammatory disease (PID) [3,4]. PID comprises a spectrum of diseases that include salpingitis, endometritis and tubo-ovarian sepsis. Typically, the pain is lower intense than in appendicitis and is bilateral. A history of vaginal discharge, dysmenorrhea and burning pain on micturition is a helpful differential diagnostic point. The physical findings include cervical tenderness on vaginal examination. When suspected, a high vaginal swab for Chlamydia trachomatis and Neisseria gonorrhoeae culture should be taken and a transvaginal ultrasound can be performed.

Table 2.

\begin{tabular}{|c|c|c|}
\hline & $\begin{array}{c}\text { Alvarado } \\
\text { (MANTRELS) } \\
\text { Score }\end{array}$ & AIR Score \\
\hline \multicolumn{3}{|c|}{ Symptoms } \\
\hline - Migratory RIF pain & 1 & \\
\hline - Anorexia & 1 & \\
\hline - Nausea or vomiting & 1 & \\
\hline - Vomiting & & 1 \\
\hline \multicolumn{3}{|c|}{ Signs } \\
\hline - Tenderness (RIF) & 2 & 1 \\
\hline - Rebound tenderness & 1 & \\
\hline - Light & & 1 \\
\hline - Medium & & 2 \\
\hline - Strong & & 3 \\
\hline - Elevated temperature $\left(>37,5^{\circ} \mathrm{C}\right)$ & 1 & \\
\hline - Elevated temperature $\left(>38,5^{\circ} \mathrm{C}\right)$ & & 1 \\
\hline \multicolumn{3}{|l|}{ Laboratory } \\
\hline - Leucocytosis & 2 & \\
\hline - $10-14,9 \times 10^{9} / \mathrm{L}$ & & 1 \\
\hline$\cdot>15 \times 10^{9} / \mathrm{L}$ & & 2 \\
\hline \multicolumn{3}{|l|}{ - Polymorphonuclear leucocytes } \\
\hline • $70-84 \%$ & & 1 \\
\hline$\bullet>85 \%$ & & 2 \\
\hline - Shift to left & 1 & \\
\hline \multicolumn{3}{|l|}{ - C-reactive protein } \\
\hline - $10-49 \mathrm{~g} / \mathrm{L}$ & & 1 \\
\hline$\bullet>50 \mathrm{~g} / \mathrm{L}$ & & 2 \\
\hline Total & 10 & 12 \\
\hline
\end{tabular}

Treatment is usually a combination of ofloxacin and metronidazole for 14 days. The diagnosis of PID is both difficult and important as more than $60 \%$ of women who have severe PID or multiple PID episodes become infertile. In addition, missed or delayed diagnosis are associated with chronic pelvic pain and ectopic pregnancy [5,6]. Appendicitis and pelvic inflammatory disease (PID) are two of the most common causes of abdominal pain in women of childbearing age [7]. The estimated prevalence of self-reported lifetime PID was $4.4 \%$ in sexually experienced women of reproductive age (18-44 years) in the US. Based on NHANES 2013-2014 data, an estimated 2.5 million women aged 18-44 years in the United States reported a lifetime history of PID diagnosis. Nevertheless, in the United States, 250,000 cases of appendicitis are reported annually, representing 1 million patient- 
days of admission and occurs in $7 \%$ of the US population, with an incidence of 1.1 cases per 1000 people per year [8]. Due to the similar presentations of these conditions, the rate of misdiagnosis is high. In fact, the most frequent reason for malpractice claims against emergency department physicians is missed appendicitis diagnosis. It is important to be vigilant evaluating and treating women at risk for appendicitis and PID because the sequelae of either condition left untreated can be as severe as death.

The aim of this article is to review the literature to identify the criteria to distinguish appendicitis from PID, to make an earliest possible diagnosis and treatment.

\section{Material and Methods}

We conducted a review of the PubMed database searching the terms "appendicitis" and "pelvic inflammatory disease OR PID" in publications from 2000 to 2021 and filtering the results by population of women of childbearing age (13-44 years). Our search showed 95 results. After analyzing the scientific articles, we extrapolated 26.

\section{Results}

\section{Clinical Diagnosis}

Abdominal pain in women of childbearing age can be difficult to diagnose. In attempting to differentiate appendicitis from PID, many aspects of history, physical examination and clinical data can help to clarify the diagnosis. However, some of the data historically used in differentiating appendicitis and PID have not proven to be useful in studies. Women of childbearing age who go to the emergency room for acute abdominal pain usually have various symptoms that can direct the diagnosis towards a gynecological or intestinal pathology. It is usually difficult to distinguish acute appendicitis from PID just based on the patient's clinical examination during the first visit. Various studies have analyzed aspects of differential diagnosis between the two diseases. Charveriat, et al. [9] concluded that adnexal pain resulting from cervical motility is the most important clinical sign of PID (sensitivity 95-99\%) and may be associated with other symptoms such as fever, leucorrhea and metrorrhagia that support the first diagnostic suspicion. Therefore, a patient with such symptoms should be directed to a gynecological consultation and a thorough pelvic examination.

Furthermore, a positive history for sexually transmitted diseases must be investigated to support the diagnosis of PID (with a specificity of $82 \%$ ). Other authors [10] consider mandatory for a woman of childbearing age with non-specific abdominal symptoms to routinely perform both a complete abdominal examination and a pelvic-gynecological examination. Kruska [11] in 2010 summarized the symptoms of PID and acute appendicitis. Positive predictive values ratio for acute appendicitis were 8.4 for pain in the right iliac fossa, 3.6 for pain migrating from the periumbilical region to right iliac fossa, 3.2 for fever and psoas sign, and only 2.0 for positive Blumberg sign. Regarding PID, leucorrhea (positive predictive value $3,3)$ and bilateral pelvic abdominal pain $(2,5)$ were very important, while fever and leukocytosis were less specific. Dahlberg, et al. [12] specifically analyzed the clinical presentations of the two diseases and documented that although cervical and uterine pain are more specific in PID, they are still present in $28 \%$ of women with acute appendicitis. Furthermore, almost $50 \%$ of the women who had an intraoperative diagnosis of acute appendicitis did not present the classic symptoms of the disease [13]. However, another study point out that cervical motion tenderness alone is not specific for PID; it is present in $25 \%$ of cases of appendicitis and $50 \%$ of ectopic pregnancies.

Morishita study in 2007 [14] is the most relevant on the clinical presentation of these two pathologies. In this retrospective study, the data of 181 women with abdominal pain were analyzed, 109 with appendicitis diagnosis and 72 with PID. The authors demonstrated that women with PID were younger ( $p=0.003)$, with a higher body temperature ( $p=0.016)$, with bilateral pelvic pain $(p<0.001)$, and with cervical motility pain $(\mathrm{p}<0.001)$. Contrariwise, women with acute appendicitis had more epigastric pain ( $\mathrm{p}<0.001)$, migrating pain ( $\mathrm{p}<0.001)$, appetite loss ( $\mathrm{p}<0.001)$, nausea and vomiting ( $\mathrm{p}$ $<0.001)$ and right iliac fossa pain $(p<0.001)$. Other studies have focused their attention on the need of further radiological and clinical investigations to distinguish PID from acute appendicitis because the clinical examination alone is not sufficient for the correct diagnostic classification. Moreover, Dahlberg, et al. [12] analyzed the patient's number of pain episodes and their duration. Typically, an acute onset pain is associated with appendicitis than PID. Acute appendicitis' pain usually lasts 24 to 36 hours, while the pain of PID can last for weeks or until treated. It is clear how the clinic itself can direct the surgeon towards a differential diagnosis between PID and acute appendicitis in women of childbearing age, but it must be accompanied by further laboratory and radiological investigations.

\section{Laboratory Diagnosis}

All women of childbearing age who present to the emergency room with acute abdominal pain undergo a blood sample for routine blood chemistry tests. These consist of blood counts with leukocyte formula, liver function tests, renal function tests and measurement of inflammation markers (for example erythrocyte sedimentation rate and CRP). Furthermore, if the painful symptoms affect the lower quadrants of the abdomen, a chemical-physical examination of the urine could be performed [15]. From the studies published on this topic, the simple execution of routine blood tests does not allow us to differentiate PID and acute appendicitis because an increase of inflammation indices (white blood cell counts and elevated CRP) is possible in both clinical conditions. In case of prolonged observation of the patient, it may be useful 
to repeat the routine blood tests because a rapid worsening of the indexes of inflammation can highlight an acute appendicitis rather than a PID [12].

In patients of childbearing age with abdominal pain, a b-hcg test should be always done to exclude an unrecognized or ectopic pregnancy [12]. Second level examinations may be useful in patients with non-specific abdominal symptoms. Among these, certainly important is a cervical swab to diagnose genital infections (for example Neisseria gonorrhoeae or Chlamydia trachomatis) which would support the diagnosis of PID. The findings of 10 or more polymorphonuclear (PMN) leukocytes on a gram-stained endocervical smear is diagnostic of mucopurulent cervicitis and can help substantiate the diagnosis of PID. Rapid tests for the detection of these microorganisms are available [11]. Most physicians are now using nucleic acid amplification tests (NAATs) to detect chlamydia and gonorrhea. These tests amplify and detect DNA and RNA sequences and are much more sensitive than previous chlamydia and gonorrhea tests. Urine NAATs have sensitivities and specificities like those of cervical samples [12].

Newer evidence documents that microorganisms associated with bacterial vaginosis have been found in women with laparoscopically proven PID. However, it is important to realize that the sensitivity of the clinical diagnosis for PID based on a pelvic examination alone is only $60 \%$ to $70 \%$. Considering additional biochemical markers that may aid in the differential diagnosis between PID and acute appendicitis, some authors have considered intrauterine nitric oxide measurement [16]. In this retrospective case-control study, intrauterine nitric oxide (NO) values were compared in women with a laparoscopic diagnosis of appendicitis or PID. In patients with PID, NO values were found much higher than those with appendicitis or compared to healthy controls. Other biochemical markers of inflammation like interleukin-6, IL-8 and CD-64 have been associated with appendicitis compared with nonsurgical causes of acute abdominal pain [17]. On the contrary the plasma levels of LRG1 were not useful to differentiate between acute appendicitis and pelvic inflammatory disease [18].

\section{Radiological Diagnosis}

All women who come to the emergency room for acute abdominal pain undergo to first-level radiological investigations $[19,20]$. Among these, the first is abdominal ultrasound (US). This gives informations about the presence of free abdominal fluid, free air, intestinal obstruction, parenchymal pathologies (hepatic, splenic, renal, adrenal, pancreatic), appendicular and gynecological pathologies or conditions (adnexal cysts, ovarian torsions, pelvic abscesses, uterine fibroids, etc.) and vascular diseases (aneurysms or arterial dissection).

\section{Abdominal Ultrasound (US)}

In the differential diagnosis between PID and acute appendicitis, abdominal ultrasound can guide the diagnosis but, in some cases, this is not diagnostic because both clinical conditions may have similar presentation (fluid in Douglas and ovaries, salpingi and appendix edematous and thickened) [21]. Therefore, in women of childbearing age with doubtful diagnosis it is always indicated to perform a gynecological examination with transvaginal ultrasound that permit a more accurate visualization of the adnexa and the uterus. Other studies [22,23] have shown that ultrasound, in the diagnosis of acute appendicitis, has a sensitivity of $75-90 \%$ and specificity of $85-98 \%$ with the advantages of a lack of ionizing radiation and a high positive predictive value (95\%). The normal appendix is rarely seen by US. Usually, the ultrasound presentation corresponds to an edematous appendix with thickened walls, with a periappendicular fluid flap and, in the most severe cases, periappendicular air bubbles or free abdominal air. Nevertheless, the diagnosis of acute appendicitis can be made by demonstration of a non-compressible, aperistaltic, blind-ending tubular structure measuring greater than $6 \mathrm{~mm}$ in diameter. The periappendiceal fat may be echogenic due to the inflammation, and color doppler US may be helpful in showing hyperemia within the thickened wall [24].

Some findings, such as submucosal ring, echogenic fat and intraluminal gas are always absent in acute PID. Similarly, cogwheel sign or incomplete septum were not observed in appendicitis [23]. Difficulties in diagnosis occur if there is a true retrocecal appendix, if the inflammation is limited to the tip of the appendix, if gas in the appendix makes it difficult to differentiate from terminal ileum, and in perforation when the appendix decompresses effectively destroying the most important sonographic feature. Secondary findings then become necessary to assist diagnosis and include loculated pericecal fluid, thickening of adjacent bowel walls, thickening and increased echogenicity in the adjacent mesentery or omentum, and frank abscess formation. However, these latter findings are not specific for appendicitis and can be seen with other intraabdominal inflammatory processes [23]. Moreover, PID tends to be a bilateral process and sonographic features are variable related to the extent of the disease. Endometrial thickening or fluid in the appropriate clinical setting is supportive of an endometritis. Purulent fluid accumulating in the pouch of Douglas in more extensive disease can be identified sonographically as particulate fluid. Salpingitis with tubal occlusion may result in a pyosalpinx.

\section{Computer Tomography (CT)}

When the clinical presentation is non-specific and the ultrasound is non-diagnostic, patients undergo abdomen computer 
tomography (CT) with contrast medium $[19,20,22]$. The use of CT, which is the test of choice for suspected appendicitis in adults, has been shown to improve diagnostic accuracy. CT decreases the falsenegative rate to $4 \%$ overall and $8.3 \%$ in females of childbearing age. Findings on CT are overall swelling $(>6 \mathrm{~mm})$, wall thickening, and enhancement of the appendix. Secondary signs include periappendiceal fat stranding, pericolic fluid, and focal thickening of the cecum. The appendix may be difficult to visualize in cases of appendiceal rupture, which may be suspected if there is a focal defect in the wall of the inflamed appendix, an appendicolith outside the appendix, a periappendiceal fluid collection, or extraluminal gas near the appendix [20]. In Hentour's retrospective study of the role of CT in the differential diagnosis between PID and acute appendicitis, it was found that only two CT findings (appendiceal diameter and left tubal thickening) were very accurate in differentiating PID from acute appendicitis.

Consequently, the first step in interpreting abdominopelvic CT in non-appendectomised women of reproductive age with acute lower abdominal pain is to measure the appendiceal diameter. If the appendiceal diameter is $<7 \mathrm{~mm}$, appendicitis is very improbable and thus CT findings of PID should be investigated. If the appendiceal diameter is $\geq 7 \mathrm{~mm}$, left tubal thickening should be analyzed, since a left tubal diameter $\geq 10 \mathrm{~mm}$ would indicate PID rather than appendicitis [22]. Conversely, it has been shown that in PID the most present CT radiological features are haziness of the pelvic fat, obscuration of the pelvic fascial planes, thickening of the uterosacral ligaments, abnormal endometrial enhancement with fluid in the endometrial cavity, enhancement and thickening of the fallopian tubes, and abnormal enhancement, enlargement, and reactive polycystic change of the ovaries [25]. Moreover, the presence of an appendicolith, cecal origin of the tubular structure, and the presence of cecal wall thickening ("cecal bar" sign) are helpful distinguishing features of appendicitis [22]. The sensitivity of CT in appendicitis is from $87 \%$ to $98 \%$, higher than US o MRI.

\section{Nuclear Magnetic Resonance (NMR)}

In some centers, especially in the United States, the use in emergency of nuclear magnetic resonance (NMR) has become routine in the management of acute abdominal pain in women of childbearing age and in pregnant women [26,27]. Magnetic Resonance Image (MRI) is a valuable tool in the evaluation of suspected appendicitis, either as an adjunct to or a replacement for an initial right lower quadrant US. This is particularly true for pediatric and pregnant patients, in whom ionizing radiation must be avoided. Scan times have been decreased using protocols including as few as four sequences, which allow for diagnostic imaging of the abdomen and pelvis in almost 15 minutes [21]. MRI has sensitivity, specificity, and accuracy for diagnosis of appendicitis of $95 \%$, 89\%, and 93\%, respectively. For diagnosis of appendiceal perforation, MRI imaging demonstrates slightly higher sensitivity
(57\%) than ultrasound with conditional CT (48\% sensitivity) [22]. The inflammation will be depicted best on the fluid-sensitive T2weighted images; fat saturation allows the signal abnormality to be more readily apparent. Appendiceal wall thickening $(>2 \mathrm{~mm})$ will show hypointense signal on T1-weighted images and hyperintensity on T2-weighted images, with the caliber of the appendix measuring greater than $6 \mathrm{~mm}$.

Periappendiceal fat inflammation will manifest as hyperintense T2 signal, consistent with edema. In the nonpregnant patient, gadolinium contrast administration increases the sensitivity of MRI in diagnosing acute appendicitis where intense appendiceal wall enhancement is seen on the fat-suppressed postcontrast T1 images [28]. MRI imaging, given the lack of a radiation-associated risk and the relative safety of gadolinium- based contrast agents, is an attractive alternative to CT for the evaluation of pelvic inflammatory disease. Compared with US, MRI imaging provides superior depiction of adnexal edema. T2-weighted fat-saturated images show parametrial signal hyperintensity due to edema. A tuboovarian abscess appears with mild signal hyperintensity on T1weighted images and high signal intensity on T2-weighted images be- cause of internal debris. At contrast-enhanced MR imaging, an abscess has a thick enhancing wall. Occasionally, an adjacent fluidfilled tubular structure, which represents an inflamed fallopian tube, is seen: hydrosalpinx, which may manifest with acute lower abdominal pain, is well depicted at MR imaging.

\section{Discussion}

PID and acute appendicitis remain two frequent pathologies in women of childbearing age and for this reason many studies have been performed on this topic. The differential diagnosis between the two pathologies may not be easy, especially in vague clinical presentation and with doubtful symptoms. The general surgeon must pay attention to the possible non-intestinal causes of acute abdominal pain in young women, also excluding all those gynecological causes that can mimic acute appendicitis. Studies have shown that in these patients a complete physical examination must always include a gynecological examination with cervicovaginal swabs to look for possible infections. Furthermore, an adequate sexual history should always be investigated as well as the use of intrauterine devices that can cause PID. The most recent studies have shown how a careful clinical analysis, before laboratory and radiological tests, can guide towards the right diagnosis. Pain migration from periumbilical to right iliac fossa with positive Blumberg's sign is more pathognomonic of acute appendicitis than bilateral pain, lasting more than 24-36 hours and characterized by non-worsening that are more specific in PID.

Routine laboratory investigations (complete blood count, leukocyte count, CRP, erythrocyte sedimentation rate) do not help to define a correct diagnosis because they are non-specific 
indicators of inflammation. Microbiological investigations would be more appropriate to investigate sexually transmitted infections (Neisseria gonorrhea, Chlamydia tracomatis) in young women with non-specific abdominal symptoms. More specific laboratory tests such as the search for inflammatory interleukins 6 and 8 or the measurement of intrauterine nitric oxide levels appear to be too specific investigations to be part of routine clinical practice and should be reserved within study protocols. Among the radiological investigations, ultrasound is certainly the first diagnostic step in young women of childbearing age with acute abdominal pain. This easily gives information in severe clinical presentation of abdominal pathology but is unfortunately little diagnostic in ambiguous cases with non-specific symptoms. CT appears to be the most specific and sensitive diagnostic test, with the best diagnostic accuracy in distinguishing between PID and acute appendicitis.

However, it should be avoided in children and pregnant women. CT images have the advantage of defining the characteristics of PID and acute appendicitis more specifically. The dimensional cut-off on the thickness of the appendicular walls of 6-7 $\mathrm{mm}$ is universally accepted as a pathognomonic finding of acute appendicitis. As well as the thickening of the uterosacral ligaments, abnormal endometrial enhancement with fluid in the endometrial cavity, enhancement and thickening of the fallopian tubes, and abnormal enhancement, enlargement, and reactive polycystic change of the ovaries are more specific radiological signs in PID. MRI plays a secondary role because it can be rarely performed in emergency, except in hospitals with great economic and technological resources. The advantage is no exposition to radiation of patients and excellent specificity and sensitivity in best defining acute appendicitis or PID. However, it should be reserved for specific population groups (pregnant women, children).

On the other hand, transvaginal ultrasound is a relatively simple and inexpensive diagnostic test that could easily complement the classic abdominal US and be part of the routine diagnostic protocol of women with acute abdominal pain. This radiological test has good sensitivity and specificity to exclude acute appendicitis in those cases where the gynecological cause of pain is more likely. Certainly, new and further studies are needed to define the best diagnostic protocol in women of childbearing age with acute abdominal pain presenting in ED. Above all, prospective multicenter studies or randomized trials would be needed.

\section{Conclusion}

PID and acute appendicitis are two very frequent pathologies in women of childbearing age. A correct differential diagnosis may not always be easy, especially in not typically clinical presentation. Therefore, a careful management of the patient starting from the clinical examination and passing through the laboratory and radiological characteristics is necessary. A correct diagnosis allows a better patient management and reduction of unnecessary surgeries.

\section{References}

1. LG Graff IV, D Robinson (2001) Abdominal pain and emergency department evaluation. Emergency Medicine Clinics of North America 19(1): 123-136.

2. S Di Saverio, Podda M, De Simone B, Ceresoli M, Augustin G, et al. (2020) Diagnosis and treatment of acute appendicitis: 2020 update of the WSES Jerusalem guidelines. World Journal of Emergency Surgery 15(1): 27.

3. Prystowsky JB, Pugh CM, Nagle AP (2005) Appendicitis. Current Problems in Surgery 42(10): 694-742.

4. Silen W, Cope Z (2010) Cope's Early Diagnosis of the Acute Abdomen. Oxford University Press, United Kingdom.

5. Weström L (1980) Incidence, prevalence, and trends of acute pelvic inflammatory disease and its consequences in industrialized countries. American Journal of Obstetrics and Gynecology 138(7) 880-892.

6. Westrom L, Bengtsson LP, Mardh PA (1981) Incidence, trends, and risks of ectopic pregnancy in a population of women. BMJ 282(6257): 15-18.

7. (2017) Prevalence of Pelvic Inflammatory Disease in Sexually Experienced Women of Reproductive Age-United States, 2013-2014. Annals of Emergency Medicine 70(1): 28-30.

8. Craig S (2021) Appendicitis: Practice Essentials, Background, Anatomy. Medscape.

9. Charvériat A, Fritel X (2019) Diagnostic d'une infection génitale haute : critères cliniques, paracliniques, imagerie, et cœlioscopie. RPC infections génitales hautes CNGOF et SPILF. Gynécologie Obstétrique Fertilité \& Sénologie 47(5): 404-408.

10. Lloyd TDR, Malin G, Pugsley H, Garcea A, Garcea G, et al. (2006) Women presenting with lower abdominal pain: A missed opportunity for chlamydia screening?. The Surgeon 4(1): 15-19.

11. Kruszka P, Kruszka SJ (2021) Evaluation of Acute Pelvic Pain in Women. American Family Physician 82(2): 141-147.

12. Dahlberg DL, Lee C, Fenlon T, Willoughby D (2004) Differential diagnosis of abdominal pain in women of childbearing age. Appendicitis or pelvic inflammatory.

13. Amit K Bhavsar, Gelner EJ, Shorma T (2021) Common Questions About the Evaluation of Acute Pelvic Pain - PubMed. American family physician 93(1).

14. Morishita K, Gushimiyagi M, Hashiguchi M, Stein GH, Tokuda Y (2007) Clinical prediction rule to distinguish pelvic inflammatory disease from acute appendicitis in women of childbearing age. The American Journal of Emergency Medicine 25(2): 152-157.

15. Reust CE, Williams A (2021) Acute Abdominal Pain in Children PubMed, American family physician 93(10).

16. Sioutas A, Ehrén I, Lundberg JO, Wiklund NP, Gemzell-Danielsson (2008) Intrauterine nitric oxide in pelvic inflammatory disease. Fertility and Sterility 89(4): 948-952.

17. Saito JM (2012) Beyond appendicitis. Current Opinion in Pediatrics 24(3): 357-364.

18. Lontra MB, Savaris RF, Cavazzola LT, Maissiat J (2021) Comparison of leucine-rich alpha-2-glycoprotein-1 (LRG-1) plasma levels between patients with and without appendicitis, a case-controlled study. Scientific Reports 11(1).

19. Heiken JP, Katz DS, Menu Y (2021) Emergency Radiology of the Abdomen and Pelvis: Imaging of the Non-traumatic and Traumatic Acute Abdomen. IDKD Springer Series: 123-143. 
20. El Hentour K, Millet I, Pages Bouic E, Curros Doyon F, Molinari N, et al. (2017) How to differentiate acute pelvic inflammatory disease from acute appendicitis? A decision tree based on CT findings. European Radiology 28 (2): 673-682.

21. Wongwaisayawan S, Kaewlai R, Dattwyler M, Abujudeh HH, Singh AK (2016) Magnetic Resonance of Pelvic and Gastrointestinal Emergencies. Magnetic Resonance Imaging Clinics of North America 24(2): 419-431.

22. Vandermeermd FQ, Wong You Cheong JJ (2010) Imaging of Acute Pelvic Pain. Topics in Magnetic Resonance Imaging 21 (4): 201-211.

23. Sivyer P (2000) Pelvic Ultrasound in Women. World Journal of Surgery 24(2): 188-197.

24. Molander MVP, Paavonen J, Sjöberg J, Savelli L, Cacciatore B (2002) Transvaginal sonography in the diagnosis of acute appendicitis. Ultrasound in Obstetrics and Gynecology 20(5): 496-501.

ISSN: 2574-1241

DOI: 10.26717/BJSTR.2021.35.005738

Perrone Gennaro. Biomed J Sci \& Tech Res

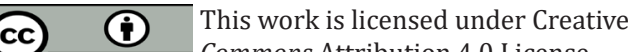

Submission Link: https://biomedres.us/submit-manuscript.php
25. Revzin, Mathur M, Dave HB, Macer ML, Spektor M (2016) Pelvic Inflammatory Disease: Multimodality Imaging Approach with ClinicalPathologic Correlation. RadioGraphics 36(5): 1579-1596.

26. Byott S, Harris I (2016) Rapid acquisition axial and coronal T2 HASTE MR in the evaluation of acute abdominal pain. European Journal of Radiology 85(1): 286-290.

27. Mervak BM, Wilson SB, Handly BD, Altun E, Burke LM (2019) MRI of acute appendicitis. Journal of Magnetic Resonance Imaging 50(5): 13671376.

28. Singh A, Danrad R, Hahn PF, Blake MA, Mueller PR, et al. (2007) MR Imaging of the Acute Abdomen and Pelvis: Acute Appendicitis and Beyond. RadioGraphics 27(5): 1419-1431.

\begin{tabular}{lll}
\hline BIOMEDICAL & Assets of Publishing with us \\
\hline RESEARCHES & $\bullet$ Global archiving of articles \\
\hline & - Immediate, unrestricted online access \\
\hline
\end{tabular}

